\title{
List of Authors
}

\section{Emmanuel Alloa}

Universität Basel

eikones / NFS Bildkritik

Rheinsprung 11

4051 Basle

Switzerland

emmanuel.alloa@unibas.ch

\section{Peter Berz}

Humboldt-Universität zu Berlin

Institut für Kulturwissenschaft

Unter den Linden 6

10099 Berlin

Germany

peter.berz@cms.hu-berlin.de

\section{Franz Josef Czernin}

8674 Rettenegg

Austria

fjczernin@netway.at

\section{Whitney Davis}

University of California at Berkeley

Department of History of Art

415 Doe Library

Berkeley,

CA 94720

U.S.A.

wmdavis@berkeley.edu 


\section{Hana Gründler}

Kunsthistorisches Institut in Florenz

Max-Planck-Institut

Via Giuseppe Giusti 44

50121 Florenz

Italy

gruendler@khi.fi.it

\section{Richard Heinrich}

Universität Wien

Institut für Philosophie

Universitätsstraße 7

1010 Wien

Austria

richard.heinrich@univie.ac.at

\section{Herbert Hrachovec}

Universität Wien

Institut für Philosophie

Universitätsstraße 7

1010 Wien

Austria

herbert.hrachovec@univie.ac.at

\section{Markus Klammer}

Universität Basel

eikones / NFS Bildkritik

Rheinsprung 11

4051 Basle

Switzerland

markus.klammer@gmail.com 
Martin Kusch

Universität Wien

Institut für Philosophie

Universitätsstraße 7

1010 Wien

Austria

martin.kusch@univie.ac.at

\section{Marie McGinn}

University of East Anglia

Department of Philosophy

Arts Building 3.64

Norwich NR4 7TJ

United Kingdom

m.mcginn@uea.ac.uk

\section{Dieter Mersch}

Universität Potsdam

Campus Neues Palais

Am Neuen Palais 10

14469 Potsdam

dmersch@uni-potsdam.de

\section{Arley Ramos Moreno}

Universidade Estadual de Campinas

Instituto de Filosofia e Ciências Humanas (IFCH)

Caixa Postal 6110

13081-970 Cidade Universitária "Prof. Zeferino Vaz"

Barão Geraldo - Campinas

São Paolo

Brasil

arley@unicamp.br 


\section{Elisabeth Nemeth}

Universität Wien

Institut für Philosophie

Universitätsstraße 7

1010 Wien

Austria

elisabeth.nemeth@univie.ac.at

\section{Stefan Neuner}

Universität Basel

eikones / NFS Bildkritik

Rheinsprung 11

4051 Basle

Switzerland

neuner.stefan@unibas.ch

\section{Kristóf Nyíri}

Institute for Philosophical Research

Hungarian Academy of Science

Szemere u. 10

Budapest, 1054

Hungary

nyiri@hunfi.hu

Dawn M. Phillips

St Anne's College

University of Oxford

Woodstock Road

Oxford, OX2 6HS

United Kingdom

dawn.phillips@warwick.ac.uk 
Wolfram Pichler

Universität Wien

Institut für Kunstgeschichte

Universitätscampus Hof 9

Spitalgasse 2

1090 Wien

Austria

wolfram.pichler@univie.ac.at

Wolfgang Pircher

Universität Wien

Institut für Philosophie

Universitätsstraße 7

1010 Wien

Austria

wolfgang.pircher@univie.ac.at

\section{Genia Schönbaumsfeld}

University of Southhampton

School of Humanities

Southhampton, SO17 1BJ

United Kingdom

g.m.e.schoenbaumsfeld@soton.ac.uk

\section{Oliver Scholz}

Westfälische Wilhelms-Universität Münster

Philosophisches Seminar

Domplatz 23

48143 Münster

Germany

oscholz@uni-muenster.de 


\section{Jakob Steinbrenner}

Ludwig-Maximilians-Universtiät München

Geschwister-Scholl-Platz 1

80539 Munich

Germany

info@prof-steinbrenner.de

\section{David Wagner}

Universität Wien

Institut für Philosophie

Universitätsstraße 7

1010 Wien

Austria

david.wagner@univie.ac.at

Kendall L. Walton

University of Michigan

Department of Philosophy

Ann Arbor

MI 48109-1003

U.S.A.

klwalton@umich.edu 